\title{
Urban transportation system analysis in emergency conditions
}

\author{
F. Russo ${ }^{1} \&$ A. Vitetta ${ }^{1}$ \\ 1 Department of Computer Science, Mathematics, Electronics and \\ Transportation, Mediterranea University of Reggio Calabria, Italy.
}

\begin{abstract}
Methodologies for transportation planning in urban area when exogenous events occur and/or in emergency conditions have not received great attention in the past in literature. Models and algorithms specified and calibrated in ordinary conditions cannot be directly applied in emergency conditions. Users move on the network in different behavioural conditions and in general they are in panic behaviour, they do not know system congestion and reliability in real time because system is in exceptional condition, and the user optimum, in general, is different from the system optimum. Different models and algorithms have to be proposed in this scenario respect that proposed for simulating ordinary conditions.

In this paper general model and relative solution algorithms are proposed for simulating road urban network system in emergency conditions. Models and algorithms used for simulating system in ordinary conditions are proposed and compared. Pros and cons for the different models in different simulation conditions are discussed.
\end{abstract}

\section{Introduction}

Methodologies for transportation planning in urban area when exogenous events occur and/or in emergency conditions have not received great attention in the literature and only emergencies when a nuclear event occurs are treated [11] and in some case for building [9] and urban system [14]. In general, there is no systematic analysis of different events connected with transportation system design. Models and algorithms specified and calibrated in ordinary conditions [2], [3], [7], [12], [15] cannot be directly applied in emergency conditions. Users 


\section{Environmental Health Risk}

move on the network in different behavioural conditions, they do not know system congestion and reliability in real time, and the user optimum, in general, is different from the system optimum. Different models and algorithms have to be proposed in this scenario.

A problem which entails particular attention in terms of coordination and management is that strictly linked to general mobilization which is created in a densely populated area after a disaster (earthquake, flood, etc.) or in any kind of emergency.

Two different approaches are considered for planning co-ordination: "what if" models (system or user optimum with deterministic user behaviour) and "what to" models (optimal user distribution and departure time, optimal network design system).

In this paper a general framework is reported with specific methods and models (section 2) for analysing road urban transportation performance in emergency conditions when exogenous phenomena occur in the context of "what if" approach while a specification of "what to" model is reported in [13]. The obtained results confirm the goodness of procedure and highlight some aspects that need to develop ([17], [18], [19], [20]). Some indication about the single paper reported for models and applications is reported in section 3 .

\section{Models for the simulation in emergency condition}

The simulation in emergency condition needs to study two different models strictly connected: the risk analysis and the evacuation simulation.

\subsection{Risk analysis}

Definite an emergency event $E_{i}$ in an area $T$, the event $E_{i}$ can be happens in $T$ with different intensity level $\mathrm{L}_{\mathrm{Ei}}$. For each event a probability function $q\left(\mathrm{~L}_{\mathrm{Ei}}, \mathrm{T}\right)$ can be defined that represents the probability that and event $E_{i}$ happens, with level $\mathrm{L}_{\mathrm{Ei}}$ in $\mathrm{T}$ in a slice time $\Delta$. It is defined as:

$$
\int_{T} \int_{L_{E i}} q\left(L_{E i}, T\right) d L_{E i} d T=1
$$

The function can be obtained from the frequency that the event occurs .

The probability that an events $E_{i}$ occurs in $T$ with intensity level between $A_{E i}$ and $\mathrm{B}_{\mathrm{Ei}}$ in an time slice $\Delta$ is:

$$
\mathrm{P}_{\mathrm{Ei}, \mathrm{T} \Delta}\left(\mathrm{A}_{\mathrm{Ei}}<\mathrm{L}_{\mathrm{Ei}}<\mathrm{B}_{\mathrm{Ei}}\right)=\int_{\mathrm{T}} \int_{\mathrm{A}_{\mathrm{Ei}}}^{\mathrm{B}_{\mathrm{Ei}}} \mathrm{q}\left(\mathrm{L}_{\mathrm{Ei}}, \mathrm{T}\right) \mathrm{dL_{ \textrm {Ei } }} d \mathrm{~T}
$$

The probability that an events $E_{i}$ occurs in $T$ with intensity level upper than $C_{E i}$ in an time slice $\Delta$ is:

$$
P_{E i, T} \Delta\left(L_{E i}>C_{E i}\right)=\int_{T} \int_{A_{E i}}^{+\infty} q\left(L_{E i}, T\right) d L_{E i} d T
$$

$\mathrm{P}_{\mathrm{Ei}, \mathrm{T}, \Delta}\left(\mathrm{L}_{\mathrm{Ei}}>\mathrm{C}_{\mathrm{Ei}}\right)$ can be defined the "dangerousness" of the event $\mathrm{E}_{\mathrm{i}}$ in $\mathrm{T}$ and $\Delta$.

A quantitative level of social disutility that the event $E_{i}$ in $T$ and $\Delta$ generates is $R\left(T, L_{E i}\right)$ given by the product of: 


$$
R\left(T, L_{E i}\right)=V\left(T, L_{E i}\right) N(T)
$$

where

- the "vulnerability" $\mathrm{V}\left(\mathrm{T}, \mathrm{L}_{\mathrm{Ei}}\right)$, that is the capacity of infrastructures (building, road, bridge, ....) to resist to the event;

- the "exposure" $N(T)$, that is the "equivalent" value of people and infrastructure in $\mathrm{T}$.

A measure of the "risk" for the events $E_{i}$, in $T$, with intensity level between $A_{E i}$ and $\mathrm{B}_{\mathrm{Ei}}$, in an time slice $\Delta$, is:

$$
R I_{E i, T, \Delta}\left(A_{E i}<L_{E i}<B_{E i}\right)=\int_{T} \int_{A_{E i}}^{B_{E i}} R\left(T, L_{E i}\right) q\left(L_{E i}, T\right) d L_{E i} d T
$$

Considering an homogenous territory, a probability function constant ed equal to $\mathrm{p}^{\prime}$, a vulnerability $\mathrm{V}\left(\mathrm{T}, \mathrm{L}_{\mathrm{Ei}}\right)$ constant ed equal to $\mathrm{V}^{\prime}$, an exposure constant ed equal to $\mathrm{N}(\mathrm{T})$, the risk has the simply form:

$$
R I_{E i, T, \Delta}\left(A_{E i}<L_{E i}<B_{E i}\right)=V^{\prime} E^{\prime} P^{\prime}
$$

The risk modify the system simulation as reported in Fig. 1.

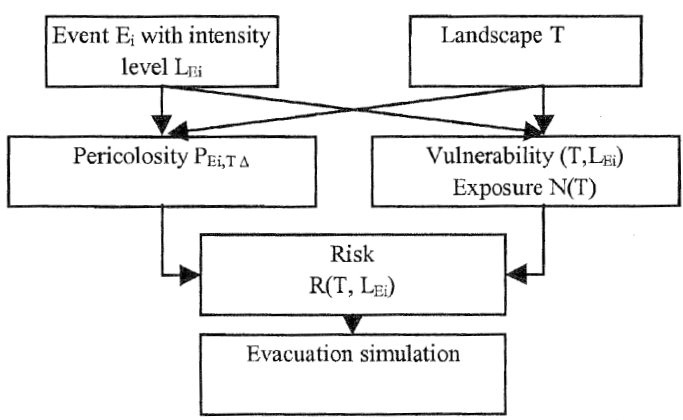

Figure 1: Relation between risk and transportation system.

\subsection{Evacuation simulation}

The drawing-up of an evacuation plan for a city requires a study of the problems connected to land use and connected to the disaster. The complete procedure for developing an evacuation plan can be subdivided into the following variously connected phases: study of the land-use; supply analysis; demand analysis; supply-demand interaction analysis; system design with "what if" and/or "what to" methodology. In Fig. 2 a system of models which may be proposed for elaborating evacuation plans and relative functional connections are represented. The demand model is subdivided into the following sub-models: Emission, with emergency or pre-arranged approach, in relation to temporal gap available between the time when the event is perceived by the public decision-maker and the time when the event occurs; Distribution towards assembly centre free (with or without user information on the system configuration) or constrained; Modal Split with different choice sets in relation to the alternatives: pedestrians towards nearby assembly centre, cars towards assembly centre further away, using special police transport modality for particular categories of disadvantaged users such as 


\section{Environmental Health Risk}

the sick, the elderly, prisoners, etc.; Route Choice free (with or without user information on the system configuration) or constrained.

The supply model is subdivided into the following sub-models: infrastructure network available for evacuation with relative characteristics; reliability level of the supply system in relation to exogenous causes.

The assignment model simulates how the transportation system works where supply and demand models are defined. The interaction model has in a static approach to consider the user objective (user deterministic or stochastic optimum) or system objective (system optimum).

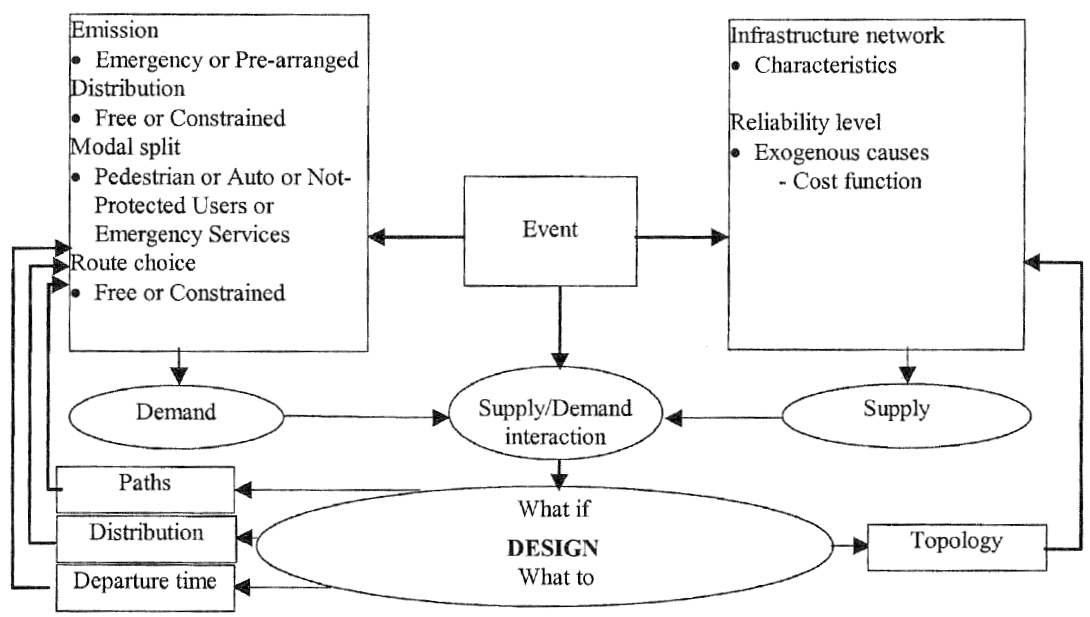

Figure 2: The global procedure for the evacuation plan and relevant links.

\subsubsection{Methods for supply analysis}

The first phase consists in studying the area as follows: the area is delimited, defined as that area comprising the transport system in question and where most of the effects of the planned interventions are believed to be; homogeneous areas are identified; zoning and minimal road network plan.

The second phase involves supply analysis which is conducted by the following: verification of the exogenous disturbances on the transport network; construction of the transport network; graph representation of the network; construction of link cost functions. All the roads are represented by links and intersections between roads with nodes. In each node the control strategy to be used must be defined.

When emergencies occur, the transport system must be optimised, chiefly to allow two operations: evacuation of system users to appropriate assembly centre; access of emergency vehicles and the security forces.

Perturbed conditions on networks may be classified in various ways [13]. As for the cause producing the disturbance, there may be traffic-endogenous and exogenous causes.

Transport supply is represented by a graph G consisting of an ordinate pair of sets, a set $\mathrm{N}$ of elements called nodes and a set $\mathrm{L}$ of pairs of nodes belonging to $\mathrm{N}$ called arcs or links: $G=(N . T)$ 
The link cost vector, $\mathbf{c}$, is defined as a vector whose generic component $\mathrm{c}_{\mathrm{ij}}$ consists of the transport cost (generalised) on link ij. To each link may be associated a link flow $\mathrm{f}_{\mathrm{ij}}$ ( $\mathrm{f}$ being the link flow vector) which represents the average number of users which, in the time unit, uses the link. The flow vector can only assume values belonging to its feasibility set denoted by $S_{\mathrm{f}}$. The scalar function $c_{i j}(\mathbf{f})$, which allows us to calculate the average transport cost of each link corresponding to a link flow vector, is called a cost function and may be either separable or non-separable. In the former case, the cost of a link depends exclusively on the flow relative to the link itself:

$$
\mathrm{c}_{\mathrm{ij}}(\mathbf{f})=\mathrm{c}_{\mathrm{ij}}\left(\mathrm{f}_{\mathrm{ij}}\right)
$$

However, in the latter case, it depends on the flow on more than one link:

$$
\mathbf{c}=\mathbf{c}(\mathbf{f})
$$

In transport networks, various functions may be used to relate travel time $\mathrm{c}$ or velocity $v$ on the link to vehicle flow $f$, capacity $C$ and other characteristics. One of the most commonly used functions is the BPR function which relates travel time to zero flow $t_{0 i j}$ of a link $\mathrm{ij}$, vehicle flow $\mathrm{f}_{\mathrm{ij}}$ on the link and capacity $\mathrm{C}_{\mathrm{ij}}$ to travel time $t_{\mathrm{ij}}$ :

$$
t_{i j}=t_{0 i j}\left[1+\alpha(f / C)^{\beta}\right]
$$

$\alpha$ and $\beta$ being parameters greater than zero to be calibrated.

For queuing at junctions Doherty's adaptation of Webster's formula is generally used.

Some indications about link reliability for motorway link is reported in [10].

\subsubsection{Methods for demand analysis}

The use of demand models in simulating an evacuation plan involves various problems connected with the many cases that may lead to a state of emergency. Below are considered two decisional units: on the one hand the citizen user who has to decide in some cases how to act, and on the other the public decisionmaker (Prefect, Mayor, etc.) who may impose or advise some user choices. The public decision-maker must predict the effects that implementation that his/her measures have on the system, and must bear in mind that his/her proposed solutions may interact with the individual reactions of the users served by the transport system in question.

Average demand, with its main characteristics in a certain reference period, may be obtained with a system of demand models, that allows us to associate to a given system of activities and transport supply the average number of trips with certain characteristics and in a reference period. In all choice models, we need to define the set of alternatives considered by the public decision-maker and the user, as well as the type of choice models considered [2], [3]. The sequence of submodels is as follows: emission model supplying trips originating from a certain zone in the study area; departure time model supplying the number of users leaving in each time slice; distribution model supplying the number of users reaching a particular assembly centre from a fixed origin zone; modal split model supplying the number of users using a given transport modality from a certain 


\section{Environmental Health Risk}

origin to a certain assembly centre; route choice model supplying the percentage of users who use a certain route between a fixed origin and assembly centre with a transport modality.

The term o is used to indicate generic trip origin and $d$ assembly centre, $d_{o d}$ being the number of users travelling between the origin and assembly centre pair $(o, d)$. The demand vector $\mathbf{d}$ (whose generic element is $\mathrm{d}_{\mathrm{od}}$ ) may assume values belonging to its feasibility set denoted by $S_{d}$. The choice model from a given choice set, in the context of random utility models, assumes that a generic user, travelling between an origin- assembly centre pair $(o, d)$, associates to each alternative $\mathrm{k}$ belonging to the set $\mathrm{K}_{\text {od }}$ of available alternatives connecting that $\mathrm{O} / \mathrm{D}$ pair, a perceived utility $\mathrm{U}_{\mathrm{k}, \mathrm{od}}$ which may be expressed as:

$$
\mathrm{U}_{\mathrm{k}, \mathrm{od}}=\mathrm{V}_{\mathrm{k}, \mathrm{od}}+\varepsilon_{\mathrm{k}, \mathrm{od}} \quad \forall \mathrm{k} \in \mathrm{K}_{\mathrm{od}}
$$

The term $\mathrm{V}_{\mathrm{k}, \mathrm{od}}$ denotes the average, or systematic, utility of alternative $\mathrm{k}$ while the random residual $\varepsilon_{\mathrm{k}, \mathrm{od}}$ is usually assumed to include perception errors of the decision maker as well as the modelling approximation of the analyst.

If the $\varepsilon_{\mathrm{k}, \mathrm{od}}$ are assumed to be independent and identical Gumbel $\mathrm{G}(0, \theta)$ variates of zero mean and parameter $\theta$ more than zero, then the well-known Multinomial Logit model of choice results [2]. The probability of choosing an alternative $\mathrm{k}$ between $o$ and $d$, giving a choice set $K_{o d}, p_{o d}\left(k / K_{o d}\right)$, is:

$$
p_{o d}\left(k / K_{o d}\right)=\exp \left(V_{k, o d}\right) / \Sigma_{h \in K o d} \exp \left(V_{h, o d}\right)
$$

If the residuals are assumed to be jointly distributed as a Multivariate Normal of zero mean, the Probit choice model is obtained [8].

Different specifications can be used for term $V_{k, o d}$. A possible specification is:

$$
V_{\mathrm{k}, \mathrm{od}}=\Sigma_{1} \alpha_{\mathrm{l}} X_{1}-\Sigma_{\mathrm{m}} \beta_{\mathrm{m}} Y_{\mathrm{m}}
$$

where $\alpha_{1}$ and $\beta_{\mathrm{m}}$ are parameters greater than zero to calibrate; $X_{1}$ are user utility attributes; $Y_{m}$ are user disutility attributes.

In Tab. 1 an example of attribute specification of the different models is reported.

Table 1. Example of attributes specification for choice models.

\begin{tabular}{lll}
\hline Choice Dimension & Utility Attributes $X_{l}$ & Disutility Atributes $Y_{m}$ \\
\hline Emission & Resident, vehicle owner, number or people & \\
& $\quad$ in family, sick & \\
Departure time & Accessibility, resident & Congestion \\
Distribution & Resident, worker, students & Distance, travel time \\
Modal split & Vehicles owner, modality available & Travel time \\
Route choice & & Travel time \\
\hline
\end{tabular}

\subsection{Methods for supply-demand interaction analysis}

A mathematical model of supply-demand interaction allows us to simulate the way in which the demand uses the supply system, producing flows on network links that in turn generate travel costs. System configuration, simulated through models, depends on user behaviour hypotheses (attributes used, distribution of random residuals, level of information available on supply, quality of information). Demand-supply interaction in the system, in the case of evacuation, cannot be tackled by using static assignment models directly with stationary flow. 
On the other hand, the use of dynamic assignment models, in a non stationary approach, is fairly complex in that there are many parameters to calibrate and there is often a lack of complete data regarding transport systems on which to calibrate the models. The main models that can be used for analysing demandsupply interaction are in the following reported.

In the stationary flow simulation approach: the stochastic equilibrium approach, i.e. the use of static assignment models with stochastic user behaviour, by which user information is more or less correctly reproduced, non-perfect respect of impositions, alternative choice behaviour; the deterministic equilibrium approach, i.e. the use of static assignment models with deterministic user behaviour, by which complete user knowledge of the system is reproduced; the system optimum approach, i.e. the use of static assignment models with user behaviour imposed by the public decision-maker, . In the case of equilibrium (stochastic or deterministic) each user tends to maximise his/her utility and minimise costs, while in the case of the system optimum, we may consider a system in which all the choices are made in such a way that the utility of all those to be evacuated is maximised. The differences between the two behaviour types (deterministic and stochastic) and the effects in terms of assignment have been analysed by Wardrop [16]. The solutions for the three models are not identical.

Stochastic equilibrium assignment is obtained in the hypothesis of probabilistic route choice with congested networks. In the literature this problem is indicated by the abbreviation SUE (Stochastic User Equilibrium). The resulting route flows $\mathbf{F}^{*}$ correspond to a condition in which, for each OD pair, the perceived cost of the routes used at equilibrium is less than or equal to the equilibrium cost perceived for every other route, and may be obtained by the following type of model:

$$
\mathbf{F}^{*}=\mathbf{P}\left(\mathbf{A}^{\mathrm{T}} \mathbf{c}\left(\mathbf{A} \mathbf{F}^{*}\right)\right) \mathbf{d} \quad \text { where } \mathbf{F}^{*} \in \mathrm{S}_{\mathrm{F}}
$$

where $\mathbf{P}$ is the probability matrix whose generic element supplies the probability of a route $\mathrm{k}$ belonging to an OD pair, $\mathbf{d}$ is the demand vector, $\mathrm{S}_{\mathrm{F}}$ is the feasibility set of route flows. Similarly, to obtain the link flows vector $\mathrm{f}^{*}$ the model is:

$$
\mathbf{f}^{*}=\mathbf{A} \mathbf{P}\left(\mathbf{A}^{\mathrm{T}} \mathbf{c}\left(\mathbf{f}^{*}\right)\right) \mathbf{d} \quad \text { where } \mathbf{f}^{*} \in \mathrm{S}_{\mathrm{f}}
$$

where $S_{\mathrm{f}}$ is the feasibility set of link flows. Conditions which ensure the existence and uniqueness of stochastic equilibrium link flows and costs are, respectively, continuity and monotonicity of cost functions (a condition not always respected in the presence of vehicle queues on the network).

Deterministic equilibrium assignment supplies the vector of link flows $\mathbf{f}^{*}$ (or similarly route flows $\mathbf{F}^{*}$ ) with the following relation:

$$
\mathbf{c}\left(\mathbf{f}^{*}\right)^{\mathrm{T}}\left(\mathbf{f}-\mathbf{f}^{*}\right) \geq 0 \quad \forall \mathbf{f} \in \mathrm{S}_{\mathrm{f}}
$$

termed "variational disequality".

The flows obtained respect Wardrop's principle according to which the users in equilibrium conditions use only least cost routes. In practice, users tend to minimise their own individual cost without considering the overall cost of users travelling on the network.

System optimum (SO) assignment models hypothesise that all users are 


\section{Environmental Health Risk}

channelled towards routes so as to obtain the minimum overall cost for all users on the whole network. Considering the behaviour of each user, the behavioural hypotheses underlying the SO are somewhat unrealistic if the user should choose a non-optimal route for him/her, but one which greatly benefits other users. In the case of evacuation, it is perfectly legitimate for the public decision-maker to seek to optimise trips globally; where emergency trips have to be planned, the use of this type of model should be mandatory. System Optimum assignment is defined by an optimisation model, expressed in terms of link flows, the objective function being total user cost:

$$
\mathbf{f}^{*}=\arg \min z(\mathbf{f})=\mathbf{f}^{\mathrm{T}} \mathbf{c}(\mathbf{f}) \quad \text { where } \mathbf{f}^{*} \in \mathrm{S}_{\mathbf{f}} .
$$

Models with a stationary approach are good at simulating systems with a uniform user flow on links in the simulation period and also with an overall demand level compatible with system capacities. In evacuation problems, such hypotheses are restrictive since the flow can be non-stationary and unstable, and the links all have a capacity lower than demand. Thus to use such models, it is worth simulating the system by subdividing simulation time into several intervals in which flow is maintained in stationary conditions and demand, to be assigned, is compatible with supply. This approach can be called pseudo-dynamic. Within the pseudo-dynamic approach, one of the three assignment models proposed in this section may be used.

Tab. 2 summarises the proposed demand models with relative specifications for the choice set generation and for choice form a given choice set, for public decision-maker and for user.

In non-stationary models [1], [4], [5], [6], it is hypothesised that the flow variables may have a variability not only in space but also in time. There may be three aggregation levels in representing the flow: aggregate flow parameters; analysis of homogeneous vehicle groups (vehicle packages); analysis of individual vehicles. Macroscopic models use a continuous representation of the flow, as if it were a fluid in a similar way to that of hydrodynamic theory. Microscopic and mesoscopic models employ a desegregate representation of individual (microscopic) or group user behaviour (mesoscopic). Model outputs either refer to the individual user or group of users. In the case of macroscopic models, the individual vehicle (or group of vehicles) is not monitored directly while in the other models vehicle trajectories are directly monitored. In evacuation problem generally the macroscopic models are not used.

Table 2 - Choice set alternatives and choice models.

\begin{tabular}{|c|c|c|c|c|}
\hline \multirow{2}{*}{$\begin{array}{c}\text { Choice } \\
\text { Dimension }\end{array}$} & \multicolumn{2}{|l|}{ Choice set } & \multicolumn{2}{|c|}{ Choice model/Assignment } \\
\hline & Public decision-maker & User & Pub. decis.-maker & User \\
\hline Emission & $\begin{array}{l}\text { Emergency (Immediately or Not evacuate) } \\
\text { Pre-arranged } \quad \text { (Delayed or Not evacuate) }\end{array}$ & $\begin{array}{l}\text { Like pub. } \\
\text { decis. maker }\end{array}$ & System optimum & $\begin{array}{l}\text { Stochastic user } \\
\text { equilibrium }\end{array}$ \\
\hline $\begin{array}{l}\text { Departure } \\
\text { time }\end{array}$ & $\begin{array}{l}\text { Constrained (Time slice } 1, \text { Time ....) } \\
\text { Free } \quad \text { (Time slice } 1, \text { Time ....) }\end{array}$ & $\begin{array}{l}\text { Like pub. } \\
\text { decis. maker }\end{array}$ & System optimum & $\begin{array}{l}\text { Stochastic user } \\
\text { equilibrium }\end{array}$ \\
\hline Distribution & $\begin{array}{l}\text { Constrained (Centre } 1, \text { Centre 2, ..) } \\
\text { Free } \quad(\text { Centre } 1, \text { Centre 2, ..) }\end{array}$ & $\begin{array}{l}\text { Like pub. } \\
\text { decis. maker }\end{array}$ & System optimum & $\begin{array}{l}\text { Stochastic user } \\
\text { equilibrium }\end{array}$ \\
\hline Modal split & Vehicle, Pedestrian, Emergency services & $\begin{array}{l}\text { Like pub. } \\
\text { decis. maker }\end{array}$ & System optimum & $\begin{array}{l}\text { Stochastic user } \\
\text { equilibrium }\end{array}$ \\
\hline Route choice & Exhaustive, Filtered & Behavioural & System optimum & Stochastic u. e. \\
\hline
\end{tabular}




\section{Structure of the models and applications}

The pseudo-dynamic, mesoscopic and microscopic models are proposed in the following papers presented in the same proceeding:

- evolution of an urban transportation system in emergency conditions: analysis through a pseudo-dynamic assignment model [17];

- use of a mesoscopic dynamic assignment model for approaching the evolution of an urban transportation system in emergency conditions [18];

- microscopic approach for the evaluation of an urban transportation system in emergency conditions [19].

The application and the results comparisons are reported in the following paper presented in the same proceeding:

- analysis and comparison of several urban road transportation assignment models [20].

Research for the analysis and the modelling of transportation systems in emergency conditions requires new studies in the supply, demand and supplydemand interaction. In emergency conditions there is the necessity to develop new methodologies and to rearrange standard procedures such as: network vulnerability analysis in terms of "security coefficient" of the supply system in relation to the events with different levels of hazards and different probabilities of fulfilment; specification and calibration of link cost functions to use in system simulation in over-saturation conditions in the analysis periods; specification and calibration of demand models for the different choice levels for users and for the public decision-maker.

\section{References}

[1] Ben Akiva, M. \& De Palma, A., Dynamic models of transportation networks. Proc. of the $15^{\text {th }}$ PTRC Summer Annual Meeting, University of Bath, England, 1987.

[2] Ben Akiva, M. \& Lerman, S., Discrete choice analysis: theory and application to travel demand. MIT Press, Cambridge, Mass, 1985.

[3] Cascetta, E. Transportation systems engineering: theory and methods. Kluwer, Academic Press, 2001.

[4] Cascetta, E. A stochastic process approach to the analysis of temporal dynamics in transportation networks. Transportation Research 23 B, 1-17, 1989.

[5] Cascetta, E. \& Cantarella, G.E. A Day to Day and Within Day Dynamic Stochastic Assignment model. Transportation Research 25 A, 277-291, 1990.

[6] Cascetta, E. \& Cantarella, G.E. Modelling dynamics in transportation networks. Transportation Science, 1993.

[7] de Luca, M., Manuale di Pianificazione dei Trasporti, Franco Angeli, 1999.

[8] Daganzo, C.F., Multinomial Probit: the theory and its applications to demand forecasting. Academic Press, New York, 1979. 


\section{Environmental Health Risk}

[9] Di Gangi, M., Longo, A. \& Polidoro, R., Una procedura di carico dinamico per la valutazione dei piani di evacuazione. Proc. of $2^{\circ}$ seminario scientifico su Metodi e Tecnologie dell'Ingegneria dei Trasporti, Università di Reggio Calabria.

[10] Ferrari, P., Freeway Capacity: Reliability And Control. In Concise Encyclopedia of Traffic and Transportation. Papageourgiou M. editor. Pergamon Press pp. 149-152, 1991.

[11] Goldblatt, R. Development of Evacuation Time Estimates for the Davis Nuclear Power Station. T. R., 1993.

[12] Ortuzar, J.deD. \& Willumsen, L.G. Modelling transport, John Wiley and Sons, 2a ed., 1994.

[13] Russo, F. \& Vitetta, A., The road network design problem to improve the safety during exogenous flow perturbations. Proc. of the $29^{\text {th }}$ ISATA, Florence, Italy, 1996.

[14] Russo, F. \& Vitetta A., Metodi per l'analisi dei sistemi di trasporto urbano in condizioni di emergenza. QD/SS n. 9, DIMET, Università di Reggio Calabria, 1999.

[15] Sheffi, Y., Urban transportation networks, Prentice Hall, Englewood Cliff, NJ, 1985.

[16] Wardrop, J.G., Some Theoretical Aspects of Road Traffic Research. Proc. Of Inst. Civil Engr. Part II, 1952.

[17] Velonà, P. \& Vitetta, A., Evolution of an urban transportation system in emergency conditions: analysis through a pseudo-dynamic assignment model. In this book, 2003.

[18] Di Gangi, M. \& Velonà, P., Use of a mesoscopic dynamic assignment model for approaching the evolution of an urban transportation system in emergency conditions. In this book, 2003.

[19] Musolino, G. \& Vitetta, A., Microscopic approach for the evaluation of an urban transportation system in emergency conditions. In this book, 2003.

[20] Di Gangi, M., Musolino, G., Russo, F., Velonà, P. \& Vitetta, A. Analysis and comparison of several urban road transportation assignment models. In this book, 2003. 